EPJ Web of Conferences 66, 06009 (2014)

DOI: $10.1051 /$ epjconf/ 20146606009

(C) Owned by the authors, published by EDP Sciences, 2014

\title{
Probing two-photon exchange with OLYMPUS
}

\author{
M. Kohl ${ }^{1,2, a}$ for the OLYMPUS Collaboration \\ ${ }^{1}$ Hampton University, Hampton, VA 23668 \\ ${ }^{2}$ Jefferson Laboratory, Newport News, VA 23606
}

\begin{abstract}
Two-photon exchange is believed to be responsible for the discrepancies in the proton electric to magnetic form factor ratio found with the Rosenbluth and polarization transfer methods. If this explanation is correct, one expects significant differences in the lepton-proton cross sections between positrons and electrons. The OLYMPUS experiment at DESY in Hamburg, Germany was designed to measure the ratio of unpolarized positron-proton and electron-proton elastic scattering cross sections over a wide kinematic range with high precision, in order to quantify the effect of two-photon exchange. The experiment used intense beams of electrons and positrons stored in the DORIS ring at $2.0 \mathrm{GeV}$ interacting with an internal windowless hydrogen gas target. The current status of OLYMPUS will be discussed.
\end{abstract}

\section{Introduction}

Measurements of the proton electric to magnetic elastic form factor ratio with the polarization transfer method at Jefferson Lab [1,2] have revealed an unexpected and large discrepancy with the elastic form factor ratio obtained using the Rosenbluth separation technique in unpolarized cross section measurements [3]. The discrepancy has been explained as the effect of hard two-photon exchange beyond the usual one-photon exchange approximation in the calculation of the elastic electron-proton scattering cross section [4-10]. The results from both methods are mostly based on the single photon exchange assumption including standard radiative corrections [11], which account for two-photon exchange only to the extent that one of the photons is soft.

Most of our understanding of the structure of the nucleon and of nuclei is based upon lepton scattering analyzed in terms of the single photon approximation, hence it is essential to precisely quantify the effect from the exchange of two and more photons. Contrary to standard radiative corrections of the lepton arm, which are calculable based on pure QED principles, calculations of the two-photon exchange amplitude are necessarily model-dependent in order to describe the intermediate off-shell hadronic state between the two photon vertices. Such calculations have been carried out e.g. in hadronic [7, 8], or partonic frameworks of generalized parton distributions and perturbative QCD $[9,10]$. Also the importance of higher-order radiative effects, not necessarily through two or more photons, has been emphasized [12].

The general structure of two-photon exchange introduces three new complex amplitudes [4]. While the imaginary parts give rise to small single-spin asymmetries, which can be measured with

\footnotetext{
a e-mail: kohlm@jlab.org
} 
transversely polarized electron beam [13], transversely polarized target, or induced transverse recoil polarization, only the real parts of the two-photon exchange amplitude are relevant for proton form factor extractions. Most calculations tend to remove the discrepancy in the form factor data, however different model assumptions generally do not lead to a consistent determination of the individual amplitudes. For a satisfactory solution for the concurrent interpretation issue of lepton scattering experiments, it is essential to definitively verify the contribution of multiple photon exchange.

The only stringent observable that directly probes the size of the two-photon exchange amplitude is the difference of unpolarized elastic electron-proton and positron-proton cross sections. Experimentally, it is more advantageous to measure the ratio of such cross sections. To leading order, the interference of the single and two-photon amplitudes yield a contribution $\propto \alpha^{3}(\alpha=1 / 137)$ to the cross section. This interference effect is odd in the number of lepton vertices and therefore changes its sign when switching between electron and positron probes. In the presence of two-photon exchange, the $e^{+} / e^{-}$cross section ratio will deviate from unity. This effect is expected to be angular dependent, to increase with the scattering angle, likewise to decrease with the virtual photon polarization $\epsilon$, and to increase with $Q^{2}$, in order to explain the form factor discrepancy. The effect disappears for $\epsilon \rightarrow 1$. Previous measurements of the ratio were carried out in the 1960's without showing significant evidence for an effect [14], as displayed in Fig. 1. Most of these data were however measured either at low $Q^{2}$ or at large $\epsilon$, where the effect is also expected to be small.

\section{The OLYMPUS Experiment}

The OLYMPUS experiment $[15,16]$ aims to precisely measure the ratio of elastic $e^{+} p$ and $e^{-} p$ scattering cross sections to better than $1 \%$ total error for a beam energy of $2 \mathrm{GeV}$ and a wide range of scattering angles. In the kinematic region covered by OLYMPUS the cross section ratio is expected to deviate from unity by as much as $5-10 \%$, if the form factor discrepancy between Rosenbluth and recoil polarization measurements is caused by two-photon exchange.

The OLYMPUS experiment has been run at the DORIS storage ring at DESY, Hamburg, Germany, which provided both electron and positron beams in excess of $100 \mathrm{~mA}$ at $2 \mathrm{GeV}$. Both the lepton and recoiling proton were detected in coincidence. The internal, unpolarized, isotopically pure hydrogen gas target was designed to deliver $3 \cdot 10^{15}$ atoms/s, corresponding to a luminosity of $2 \cdot 10^{33} /\left(\mathrm{cm}^{2} \mathrm{~s}\right)$. The OLYMPUS detector has largely been based on the previous BLAST apparatus from the MITBates Linear Accelerator Center [17-20], a toroidal spectrometer with excellent tracking capability over a wide range of scattering angles of $\approx 20^{\circ}-80^{\circ}$ and $\pm 15^{\circ}$ out of plane. Several upgrades were implemented for OLYMPUS, in particular two redundant systems were added to measure the relative luminosity of electron and positron beams using forward-angle elastic lepton-proton and symmetric Møller/Bhabha scattering, respectively. Preparations of the OLYMPUS experiment have begun upon approval in 2010 after securing the required funding from the US agencies DOE and NSF, DESY and the German agency DFG. The BLAST detector was transferred from MIT and reassembled at DESY in a park position. In summer 2011 it was brought into final position in the DORIS storage ring. The new internal hydrogen gas target has been designed and constructed at MIT with a $60 \mathrm{~cm}$ long target cell made by INFN Ferrara. The experiment was commissioned in 2011 in parallel with the regular DORIS synchrotron operation, with several dedicated beamtests before the first production data taking took place at the beginning of 2012. OLYMPUS has successfully taken data during two running periods in February 2012 and from October 2012 until January 2013. The integrated luminosity goal was exceeded with the data acquired. After the production running, detailed surveys of the target and detector geometry and of the magnetic field map were undertaken. Calibrations, data analysis and detailed simulations are now in full swing, using an integrated analysis framework. A total integrated 

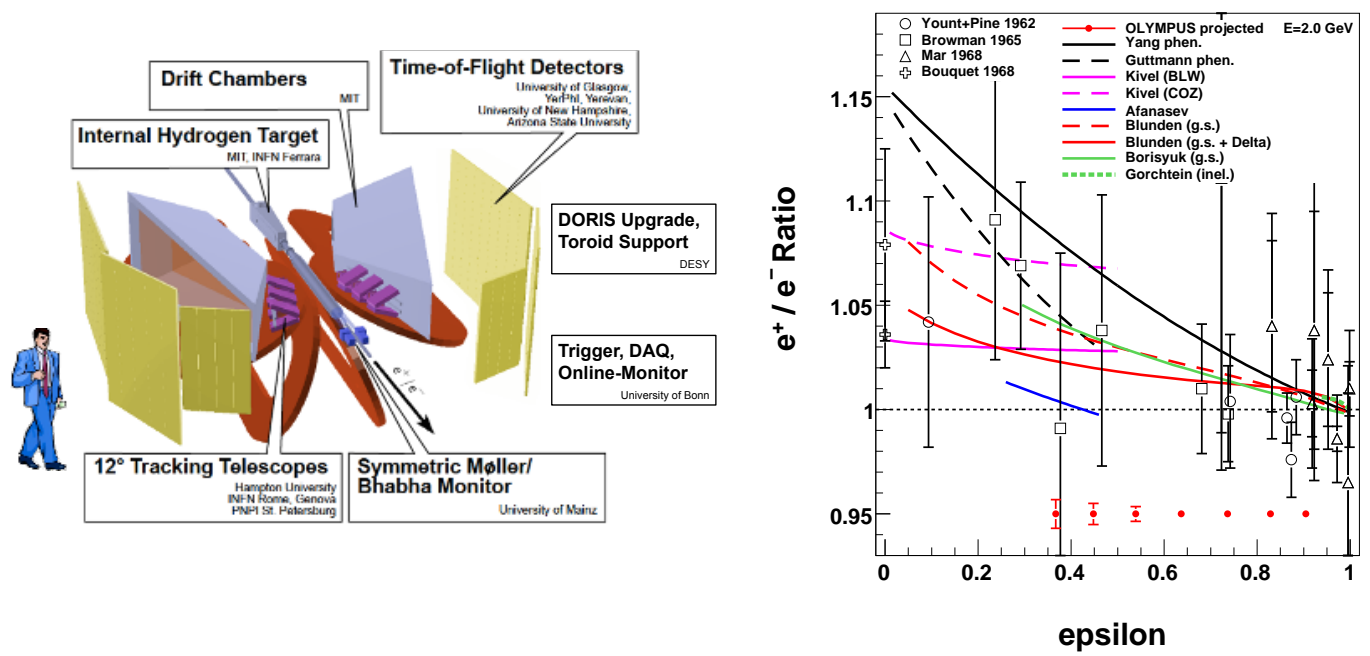

Figure 1. Left: Schematic layout of the OLYMPUS experiment with components labeled in the figure. Right: Projected precision of OLYMPUS for the $e^{+} p$ to $e^{-} p$ cross section ratio versus $\epsilon$ along with previous data [14] and various theoretical predictions [4-10], which have been evaluated for a constant beam energy of $\mathrm{E}=2 \mathrm{GeV}$.

luminosity of $3.6 \mathrm{fb}^{-1}$ at a beam energy of $2.0 \mathrm{GeV}$ is required to provide the statistical accuracy of $<1 \%$ up to $Q^{2} \leq 2.2(\mathrm{GeV} / \mathrm{c})^{2}$. The four-momentum transfer region near $Q^{2}=2.5(\mathrm{GeV} / \mathrm{c})^{2}$ is of particular interest, where the most complete experimental data set of cross sections and polarization observables exists $[2,6]$. The schematic layout of OLYMPUS is shown on the 1.h.s. of Fig 1. Of the original BLAST setup [17], the toroidal magnet, the wire chambers (WC) and the time-of-flight scintillators (TOF) have been used. Forward-angle elastic scattering luminosity monitoring systems have been constructed at Hampton University (GEM detector telescopes) and Petersburg Nuclear Physics Institute (multi-wire proportional chambers, MWPC). In addition, a symmetric Møller/Bhabha monitoring system has been developed at Mainz University. The trigger and data acquisition systems were provided by Bonn University. Figure 1 (r.h.s.) shows the projected statistical uncertainties for the $e^{+} p$ to $e^{-} p$ cross section ratio at a beam energy of $2.0 \mathrm{GeV}$ as a function of virtual photon polarization along with previous data [14] and various theoretical expectations [5-10]. The systematic uncertainties of the ratio are expected to be less than $1 \%$.

To summarize, the OLYMPUS experiment at the lepton storage ring DORIS at DESY will provide a definitive determination of the two-photon exchange effect by precisely measuring the $e^{+} p / e^{-} p$ unpolarized cross section ratio up to $Q^{2}=2.2(\mathrm{GeV} / \mathrm{c})^{2}$ and virtual photon polarization down to $\epsilon=0.37$.

The author has been supported for this work by the National Science Foundation under grants PHY-0855473, PHY-1207672, and PHY-0959521, and by DOE Early Career Award DE-SC0003884.

\section{References}

[1] A.J.R. Puckett et al., Phys. Rev. Lett. 104, 242301 (2010); V. Punjabi et al., Phys. Rev. C 71, 055202 (2005); Erratum-ibid. Phys. Rev. C 71, 069902(E) (2005) superseding M. Jones et al., 
Phys. Rev. Lett. 84, 1398 (2000); O. Gayou et al., Phys. Rev. Lett. 88, 092301 (2002); O. Gayou et al., Phys. Rev. C 64, 038202 (2001).

[2] M. Meziane et al., arXiv:1012.0339v1 [nucl-ex].

[3] I.A. Qattan et al., Phys. Rev. Lett. 94, 142301 (2005); M.E. Christy et al., Phys. Rev. C 70, 015206 (2004); R.C. Walker et al., Phys. Rev. D 49, 5671 (1994); L. Andivahis et al., Phys. Rev. D 50, 5491 (1994); G.G. Simon et al., Nucl. Phys. A333, 381 (1980); F. Borkowski et al., Nucl. Phys. A222, 269 (1974); F. Borkowski et al., Nucl. Phys. B93, 461 (1975); J.J. Murphy, Y.M. Shin, and D.M. Skopik, Phys. Rev. C 9, 2125 (1974); W. Bartel et al., Nucl. Phys. B58, 429 (1973); C. Berger et al., Phys. Lett. B35, 87 (1971); J. Litt et al., Phys. Lett. B31, 40 (1970); T. Janssens et al., Phys. Rev. 142, 922 (1966).

[4] P.A.M. Guichon and M. Vanderhaeghen, Phys. Rev. Lett. 91, 142303 (2003); M.P. Rekalo and E. Tomasi-Gustafsson, Eur. Phys. J. A 22, 331 (2004).

[5] Y.C. Chen, C.W. Kao, and S.N. Yang, Phys. Lett. B652, 269 (2007).

[6] J. Guttmann, N. Kivel, M. Meziane, and M. Vanderhaeghen, Eur. Phys. J. A 47, 77 (2011).

[7] P.G. Blunden, W. Melnitchouk, and J.A. Tjon, Phys. Rev. Lett. 91, 142304 (2003); P.G. Blunden, W. Melnitchouk, and J.A. Tjon, Phys. Rev. C 72, 034612 (2005); S. Kondratyuk, P. Blunden, W. Melnitchouk, and T.A. Tjon, Phys. Rev. Lett. 95, 172503 (2005).

[8] D. Borisyuk and A. Kobushkin, Phys. Rev. C 78, 025208 (2008); M. Gorchtein, Phys. Lett. B644, 322 (2007).

[9] Y.C. Chen, A.V. Afanasev, S.J. Brodsky, C.E. Carlson and M. Vanderhaeghen, Phys. Rev. Lett. 93, 122301 (2004); A.V. Afanasev, S.J. Brodsky, C.E. Carlson, Y.C. Chen, and M. Vanderhaeghen, Phys. Rev. D 72, 013008 (2005).

[10] N. Kivel and M. Vanderhaeghen, Phys. Rev. Lett. 103, 092004 (2009).

[11] Y.S. Tsai, Phys. Rev. 122, 1898 (1961); L.W. Mo, Y.S. Tsai, Rev. Mod. Phys. 41, 205 (1969); Y.S. Tsai, SLAC report, no. SLAC-PUB-848, 1971 (unpublished); L.C. Maximon, J.A. Tjon, Phys. Rev. C 62, 054320 (2000).

[12] Yu.M. Bystritskiy, E.A. Kuraev, E. Tomasi-Gustafsson, Phys. Rev. C 75, 015207 (2007).

[13] F. Maas, et al., Phys. Rev. Lett. 94, 082001 (2005); L. Capozza, Eur. Phys. J. A 32, 497 (2007).

[14] D. Yount and J. Pine, Phys. Rev. 128, 1842 (1962); A. Browman, F. Liu, and C. Schaerf, Phys. Rev. 139, B1079 (1965); J. Mar et al., Phys. Rev. Lett. 21, 482 (1968); B. Bouquet et al., Phys. Lett. B26, 178 (1968).

[15] A Proposal to Definitively Determine the Contribution of Multiple Photon Exchange in Elastic Lepton-Nucleon Scattering (2008); Technical Design Report for the OLYMPUS Experiment (2009); http://web.mit. edu/OLYMPUS.

[16] M. Kohl, Proc. Int. Workshop on Positrons at Jefferson Lab (JPOS2009), AIP Conf. Proc. 1160, 19 (2009); M. Kohl, Proc. 12th International Conference on Meson-Nucleon Physics and the Structure of the Nucleon (MENU 2010), AIP Conf. Proc. 1374, 527 (2011).

[17] D. Hasell et al., Nucl. Instr. and Methods in Physics Research A603, 247 (2009).

[18] C.B. Crawford et al., Phys. Rev. Lett. 98, 052301 (2007).

[19] E. Geis et al., Phys. Rev. Lett. 101, 042501 (2008).

[20] C. Zhang et al., Phys. Rev. Lett. 107, 252501 (2011). 